\title{
Random Matrix Model and the Calogero-Sutherland Model: A Novel Current-Density Mapping
}

\author{
N. Taniguchiat, B. S. Shastry ${ }^{b}$ and B. L. Altshuler ${ }^{c}$ \\ ${ }^{a}$ Department of Physics, Massachusetts Institute of Technology, Cambridge, MA 02139, USA \\ ${ }^{b}$ Indian Institute of Science, Bangalore - 560 012, India \\ ${ }^{c}$ NEC Research Institute, 4 Independence Way, Princeton, NJ 08540, USA
}

\begin{abstract}
We investigate the relation between the invariant correlators of random matrix theory and correlators of the integrable one-dimensional systems. Starting from the relation between correlators for the coupling strengths $\lambda=1 / 2,1$, and 2 , we explore the local current-density mapping applicable to arbitrary $\lambda$ including irrational values, which results from the novel structure of the CalogeroSutherland model. We find an interesting and novel relationship between equal time current and density correlations for any coupling, which exist in addition to the usual Ward Identities for this class of systems.
\end{abstract}

Suggested PACS: 05.40.+j,05.30.-d,05.45.+b

Spectral statistics of complex quantum systems such as quantum dots and quantum billiards exhibit striking universal behaviors irrespective of their microscopic details. These systems are characterized by the repulsion of energy level. To describe such spectral statistics in quantum chaotic systems, random matrix theory (RMT) and Wigner-Dyson statistics has been successfully applied [1] 3]. The universality classes of a given system is entirely determined by its generic (spin and/or time-reversal) symmetry of the system: orthogonal (spinless) and symplectic (with spin-orbit interaction) for Tinvariant systems, and unitary for T-breaking systems. Typically the level repulsion is characterized by the level spacing $\varepsilon$ distribution proportional to $\varepsilon^{\beta}$ for small $\varepsilon$, where $\beta=1,2$ and 4 respectively for the orthogonal, unitary and symplectic classes. It is worth mentioning that that the universality of Wigner-Dyson statistics corresponds to the bulk scaling limit of matrix models. It is also possible to get new and different universality classes from various edge scaling limits.

RMT is connected intimately with the CalogeroSutherland model (CSM) [1 6] which describes $N$ fermions located on a ring of the perimeter $L$. The interaction between these particles is pairwise and is inversely proportional to the square of the arc distance:

$$
H=\frac{1}{2 m} \sum_{i} p_{i}^{2}+\frac{\hbar^{2} \lambda(\lambda-1)}{m} \sum_{i<j} \frac{\phi^{2}}{\sin ^{2}\left(\phi r_{i j}\right)}-E_{0}
$$

where $r_{i j} \equiv r_{i}-r_{j}, p_{i}=-i \hbar \partial / \partial r_{i}, \phi=\pi / L, \lambda$ determines the sign and the strength of the interaction, and $E_{0}=N\left(N^{2}-1\right) \phi^{2} \lambda^{2} / 6 m$. We use the unit $\hbar=1$ hereafter.

The ground state wavefunction of Eq. (11) is given by a Jastrow function, $\psi_{0}\left(r_{1}, \cdots, r_{N}\right)=\prod_{i<j} \sin ^{\lambda}\left(\phi r_{i j}\right)$. The Jastrow form of the wave function enables ground state averages (in the thermodynamic limit) to be identified with an average over Wigner-Dyson ensembles of random matrices for coupling constants $\lambda=\beta / 2=1 / 2,1$, and 2 . In particular, the static density-density correlation function $\langle 0|\rho(r) \rho(0)| 0\rangle$ of CMS, where $\rho=\sum_{i} \delta\left(r-r_{i}\right)$ is the density operator, can be connected straightforwardly to the two-point correlator of density of states (DOS) $R_{2}(\omega)$ of RMT for these values of $\lambda$ [7].

Recently the connection between RMT and CSM has been extended from the static correlations to the dynamical correlations [8 10]. In the framework of RMT, dynamical correlations of CSM correspond to the parametric correlations depending on the external parameter. Take the random matrix which is perturbed by the external field, $H(X)=H_{0}+X \Phi$, where $H_{0}$ is a random matrix belonging to one of the Dyson ensembles, and $\Phi$ is a fixed traceless member of the same ensemble. The parametric DOS correlator $K(\Omega, X) \equiv\langle\nu(\bar{E}, \bar{X}) \nu(\bar{E}+\Omega, \bar{X}+X)\rangle$, where $\nu(E, X)=\sum_{n} \delta\left(E-E_{n}(X)\right)$ with $E_{n}(X)$ being the spectrum of $H(X)$ and $\langle\cdots\rangle$ denotes a statistical average in some interval of energy and/or external parameter, was evaluated analytically by use of the supermatrix method [11]. The resulting function $K(\Omega, X)$ is found to be universal after rescaling $\omega \equiv \Omega / \Delta$ and $x^{2} \equiv(X / \Delta)^{2}\left\langle\left(\partial E_{n}(X) / \partial X\right)^{2}\right\rangle$.

The connection with CSM is provided when we substitute $\pi \omega \rightarrow r$ and $\pi^{2} x^{2} / 2 \rightarrow-i t$ in the expression of $K(\Omega, X)$, which produces the dynamical density-density correlator of CMS $\langle 0|\rho(r, t) \rho(0,0)| 0\rangle$ for $\lambda=1 / 2,1$, and 2 . We remark that subsequently the dynamical densitydensity correlator of CMS was evaluated for arbitrary rational values of $\lambda$ by use of Jack polynomials [12].

In quantum dots or RMT, there are two kinds of two-point correlation functions which become universal [13, 14]. In terms of the retarded and advanced Green functions, these two universal functions are defined by

$$
k(\omega, x)=-\frac{1}{2}+\frac{\Delta^{2}}{2 \pi^{2}} \int d \boldsymbol{r}_{1} d \boldsymbol{r}_{2}\left\langle G_{E+\Omega, \bar{X}+X}^{R}\left(\boldsymbol{r}_{1}, \boldsymbol{r}_{1}\right) G_{E, \bar{X}}^{A}\left(\boldsymbol{r}_{2}, \boldsymbol{r}_{2}\right)\right\rangle .
$$




$$
n(\omega, x)=\frac{\Delta^{2}}{2 \pi^{2}} \int d \boldsymbol{r}_{1} d \boldsymbol{r}_{2}\left\langle G_{E+\Omega, \bar{X}+X}^{R}\left(\boldsymbol{r}_{1}, \boldsymbol{r}_{2}\right) G_{E, \bar{X}}^{A}\left(\boldsymbol{r}_{2}, \boldsymbol{r}_{1}\right)\right\rangle,
$$

where $G_{E, X}^{R, A}\left(\boldsymbol{r}, \boldsymbol{r}^{\prime}\right)=\left\langle\boldsymbol{r}\left|(E-H(X) \pm i 0)^{-1}\right| \boldsymbol{r}^{\prime}\right\rangle$ denotes the retarded and advanced Green functions. The DOS correlator is given by $K(\Omega, X)=\Delta^{-2} \operatorname{Re}[1+k(\omega, x)]$, whereas $n(\omega, x)$ has determined the response depending on the wavefunctions as well as on energy spectra.

$$
\begin{aligned}
& k_{u}(\omega, x)=\frac{1}{2} \int_{1}^{\infty} d \lambda_{1} \int_{-1}^{1} d \lambda e^{i \pi \omega\left(\lambda_{1}-\lambda\right)-\pi^{2} x^{2}\left(\lambda_{1}^{2}-\lambda^{2}\right) / 2}, \\
& n_{u}(\omega, x)=\frac{1}{2} \int_{1}^{\infty} d \lambda_{1} \int_{-1}^{1} d \lambda \frac{\lambda_{1}+\lambda}{\lambda_{1}-\lambda} e^{i \pi \omega\left(\lambda_{1}-\lambda\right)-\pi^{2} x^{2}\left(\lambda_{1}^{2}-\lambda^{2}\right) / 2},
\end{aligned}
$$

From the analytical expressions for $k(\omega, x)$ and $n(\omega, x)$, it is straightforward to observe that there is a simple differential relation connecting between these functions for all three universality classes [14]:

$$
2 \frac{\partial}{\partial x^{2}} k(\omega, x)=\frac{\partial^{2}}{\partial \omega^{2}} n(\omega, x) .
$$

The identity (5) allows us to understand what is the counterpart of $n(\omega, x)$ in the context of CSM. Indeed, by comparing Eq. (5) with the continuity relation

$$
\begin{aligned}
& \frac{\partial^{2}}{\partial t_{1} \partial t_{2}}\left\langle 0\left|\rho\left(r_{1}, t_{1}\right) \rho\left(r_{2}, t_{2}\right)\right| 0\right\rangle \\
& \quad=\frac{\partial^{2}}{\partial r_{1} \partial r_{2}}\left\langle 0\left|j\left(r_{1}, t_{1}\right) j\left(r_{2}, t_{2}\right)\right| 0\right\rangle,
\end{aligned}
$$

where $j(r)=(2 m)^{-1} \sum_{i}\left[p_{i} \delta\left(r-r_{i}\right)+\delta\left(r-r_{i}\right) p_{i}\right]$ is the current operator, we can identify $n(\omega, x)$ with $\int_{t}^{\infty}\left\langle j\left(r, t^{\prime}\right) j(0,0)\right\rangle d t^{\prime}$. Thus, according to the Kubo formula, Fourier transform of $n(\omega, x)$ with respect to $x^{2}$ gives the ac conductivity of the one-dimensional gas. The correspondence between the universal correlation functions of RMT and the correlator of CSM is summarized in Table I.

There exist another differential relation between $k(\omega, x)$ and $n(\omega, x)$ which hold only at $x \rightarrow 0$ for $\beta=1$, 2 and 4, namely,

$$
1+k(\omega, x=0)=\left.\beta \omega^{2} \frac{\partial}{\partial x^{2}} n(\omega, x)\right|_{x \rightarrow 0} .
$$

Let us first discuss some physics behind this relation. The function $n(\omega, x)$ appears when we investigate the universal properties which depend on the wavefunction as well as the spectrum. One of the examples is the dielectric response of a complex crystal with a chaotic primitive cell, where the quasi-momentum serves as the external parameter [15]. It is observed that the dynamical conductivity $\sigma(\omega)$ is exactly proportional to the DOS correlator, $1+k(\omega, 0)$ 16]. The statement can be extended to all three ensembles. This result can be physically explained as the manifestation of the independence in RMT between the fluctuations of wavefunctions (matrix elements) and those of the energy spectrum. However, the underlying theoretical structure necessary to produce $\sigma(\omega) \propto 1+k(\omega, 0)$ is highly nontrivial, since the dynamical conductivity should be formally expressed through the polarization part, hence through $n(\omega, x)$ defined by Eq. (3). We emphasize that the relation Eq. (7) remains nontrivial even when we know the explicit integral expression of $k(\omega, x)$ and $n(\omega, x)$. In fact, to see this equality explicitly by starting with the integral expressions such as Eqs. (4), we had to perform all the double$(\beta=2)$ or triple- $(\beta=1,4)$ integration by help of the Fourier transformation.

Gaining insight from Eq. (7), we expect the following equal-time relation between density-density correlator and current-current correlator will hold in the CalogeroSutherland model:

$$
\langle 0|j(r) j(0)| 0\rangle=-\frac{\lambda}{2 m^{2} r^{2}}\langle 0|\rho(r) \rho(0)| 0\rangle .
$$

We will show below that the identity Eq. (8) holds not only for $\lambda=1 / 2,1$, and 2 , but also for arbitrary values of $\lambda$, even including irrational values.

To prove Eq. (8), we fully exploit the novel structure of the Calogero-Sutherland model. The striking feature of CSM hamiltonian is that it is possessed of the factorization [17] and the supersymmetric structure 18. When we define the operator

$$
Q_{i}=p_{i}+i \lambda \phi \sum_{j \neq i} \cot \left(\phi r_{i j}\right)
$$

the Hamiltonian can be factorized as

$$
H=\frac{1}{2 m} \sum_{i} Q_{i}^{\dagger} Q_{i}
$$

where $\left[Q_{i}^{\dagger}, Q_{j}^{\dagger}\right]=\left[Q_{i}, Q_{j}\right]=0$, and 


$$
\left[Q_{i}, Q_{j}^{\dagger}\right] \equiv M_{i j}=2 \lambda \phi^{2}\left[\sum_{k \neq i} \frac{\delta_{i j}}{\sin ^{2}\left(\phi r_{i k}\right)}-\frac{\left(1-\delta_{i j}\right)}{\sin ^{2}\left(\phi r_{i j}\right)}\right] .
$$

Note that $Q_{i}|0\rangle=0$, i.e., $Q_{i}$ annihilates the ground state.

To derive the relation Eq. (8), first write the current operator in terms of operator $Q_{i}$,

$$
\begin{aligned}
\left\langle 0\left|j\left(r_{1}\right) j\left(r_{2}\right)\right| 0\right\rangle & =\frac{1}{4 m^{2}} \sum_{i j}\left\langle 0\left|\delta\left(r_{1}-r_{i}\right)\left(M_{i j}+Q_{j}^{\dagger} Q_{i}\right) \delta\left(r_{2}-r_{j}\right)\right| 0\right\rangle, \\
& =\frac{1}{4 m^{2}} \sum_{i j}\left\langle 0\left|M_{i j} \delta\left(r_{1}-r_{i}\right) \delta\left(r_{2}-r_{j}\right)\right| 0\right\rangle .
\end{aligned}
$$

As a result, we obtain

$$
\left\langle 0\left|j\left(r_{1}\right) j\left(r_{2}\right)\right| 0\right\rangle=\frac{M\left(r_{12}\right)}{4 m^{2}}\left\langle 0\left|\rho\left(r_{1}\right) \rho\left(r_{2}\right)\right| 0\right\rangle,
$$

where $M\left(r_{12}\right)=-2 \lambda \phi^{2} / \sin ^{2}\left(\phi r_{12}\right)$ for $r_{1} \neq r_{2}$. From Eq. (14), we obtain the equality of Eq. (8) in the thermodynamic limit $r_{12} \ll L$ when $M(r) \cong-2 \lambda / r^{2}$.

Using Eq. (8) and the known expression for $\langle\rho(r) \rho(0)\rangle$, we get the asymptotic behavior of the current-current correlator.

$$
\langle 0|j(r) j(0)| 0\rangle \rightarrow \begin{cases}\frac{\text { const. }}{m^{2} r^{2-2 \lambda}} & (\text { for } r \rightarrow+0) \\ -\frac{\lambda}{2 m^{2} r^{2}} & (\text { for } r \rightarrow \infty)\end{cases}
$$

We can readily extend this current-density relation to higher point correlation functions.

$$
\left\langle 0\left|j\left(r_{1}\right) \cdots j\left(r_{n}\right)\right| 0\right\rangle=\frac{1}{(2 m)^{n}}\left[\sum_{\text {all possible pairing }} M\left(r_{\alpha_{1}}-r_{\alpha_{2}}\right) \cdots M\left(r_{\alpha_{n-1}}-r_{\alpha_{n}}\right)\right]\left\langle 0\left|\rho\left(r_{1}\right) \cdots \rho\left(r_{n}\right)\right| 0\right\rangle .
$$

For instance, the four-point equal time current correlator is found to be

$$
\begin{aligned}
& \left\langle 0\left|j\left(r_{1}\right) j\left(r_{2}\right) j\left(r_{3}\right) j\left(r_{4}\right)\right| 0\right\rangle \\
& =\frac{1}{(2 m)^{4}}\left[M\left(r_{12}\right) M\left(r_{34}\right)+M\left(r_{13}\right) M\left(r_{24}\right)+M\left(r_{14}\right) M\left(r_{23}\right)\right]\left\langle 0\left|\rho\left(r_{1}\right) \rho\left(r_{2}\right) \rho\left(r_{3}\right) \rho\left(r_{4}\right)\right| 0\right\rangle, \\
& \cong \frac{\lambda^{2}}{4 m^{4}}\left[\frac{1}{\left(r_{12} r_{34}\right)^{2}}+\frac{1}{\left(r_{13} r_{24}\right)^{2}}+\frac{1}{\left(r_{14} r_{23}\right)^{2}}\right]\left\langle 0\left|\rho\left(r_{1}\right) \rho\left(r_{2}\right) \rho\left(r_{3}\right) \rho\left(r_{4}\right)\right| 0\right\rangle .
\end{aligned}
$$

The last expression is true only for the thermodynamic limit $\left(r_{i j} \ll L\right)$. We remark that for $\lambda=1 / 2$, 1 , and 2 , the $n$-point density correlators were already evaluated by Dyson within the framework of random matrix theory (2, 3), so Eq. (18) gives us a way to make an analytical evaluation of the higher-point current correlators for these values of $\lambda$. We should substitute the result for the density correlator in $N \rightarrow \infty$ limit which is presented in the form of the quaternion determinant [2]:

$$
\left\langle 0\left|\rho\left(r_{1}\right) \cdots \rho\left(r_{n}\right)\right| 0\right\rangle=\operatorname{det}\left[\sigma_{\lambda}\left(x_{i}-x_{j}\right)\right]
$$

where $1 \leq i, j \leq n ; x_{i}=k_{F} r_{i} / \pi$, and

$$
\begin{aligned}
\sigma_{1 / 2}(x) & =\left(\begin{array}{cc}
s(x) & D s(x) \\
J s(x) & s(x)
\end{array}\right), \\
\sigma_{1}(x) & =\left(\begin{array}{cc}
s(x) & 0 \\
0 & s(x)
\end{array}\right), \\
\sigma_{2}(x) & =\left(\begin{array}{cc}
s(2 x) & D s(2 x) \\
I s(2 x) & s(2 x)
\end{array}\right),
\end{aligned}
$$

where

$$
\begin{aligned}
& s(x)=\frac{\sin (\pi x)}{\pi x} ; \quad D s(x)=\frac{\partial}{\partial x} s(x), \\
& I s(x)=\int_{0}^{x} \frac{\sin \left(\pi x^{\prime}\right)}{\pi x^{\prime}} d x^{\prime} ; \quad J s(x)=I s(x)-\epsilon(x),
\end{aligned}
$$

where $\epsilon(x)$ is the function which equals to $1 / 2,0$ and $-1 / 2$, respectively, for $x>0, x=0$ and $x<0$. We note that in a very similar way, we can evaluate equal-time correlation functions of any combination of current and density operators.

In conclusion, initiating from the relation obtained from the random matrix theory, we have derived the equal-time relation between the current correlators and density correlators in the Calogero-Sutherland model, which can apply to arbitrary values of $\lambda$. General underlying structure responsible for this current-density mapping was clarified and the extension to the higher-point correlation was made. 
The authors are grateful to B. D. Simons, E. R. Mucciolo, A. V. Andreev, and A. Macedo for various useful discussions. The work of NT was supported in part by the Joint Services Electronic Program No. DAAL 03-890001 and by NSF grant No. DMR 92-14480.

* Current address: Dept. of Physical Electronics, Hiroshima University, Kagamiyama, Higashi-Hiroshima 739, Japan.

[1] F. Haake, Quantum Signatures of Chaos (SpringerVerlag, Berlin, 1991).

[2] M. L. Mehta, 'Random Matrices - Revised and Enlarged Second Edition' (Academic Press Inc., San Diego, 1991).

[3] O. Bohigas, in Chaos and Quantum Physics, edited by M.-J. Giannoni, A. Voros, and J. Zinn-Justin (NorthHolland, Amsterdam, 1991), pp. 87

[4] F. Calogero, J. Math. Phys. 10, 2191 (1969).

[5] B. Sutherland, J. Math. Phys. 12, 246 (1971).

[6] J. Moser, Adv. Math. 16, 197 (1975).

[7] B. Sutherland, Phys. Rev. B 45, 907 (1992).

[8] B. D. Simons, P. A. Lee, and B. L. Altshuler, Phys. Rev. Lett. 70, 4122 (1993); Nucl. Phys. B409[FS], 487 (1993).

[9] C. W. J. Beenakker, Phys. Rev. Lett. 70, 4126 (1993).

[10] O. Narayan and B. S. Shastry, Phys. Rev. Lett. 71, 2106 (1993).
[11] K. B. Efetov, Adv. Phys. 32, 53 (1983).

[12] Z. N. C. Ha, Phys. Rev. Lett. 73, 1574 (1994); Nucl. Phys. B435[FS], 604 (1995).

[13] M. R. Zirnbauer, Nucl. Phys. B265[FS15], 375 (1986).

[14] N. Taniguchi, A. V. Andreev, and B. L. Altshuler, Europhys. Lett. 29, 515 (1995); N. Taniguchi, B. D. Simons, and B. L. Altshuler, (unpublished).

[15] N. Taniguchi and B. L. Altshuler, Phys. Rev. Lett. 71, 4031 (1993).

[16] Though the quasimomentum is analogous to the Aharonov-Bohm flux and apparently violates $\mathrm{T}$ invariance, this invariance is recovered when the primitive cell has the reflection symmetry, as was discussed by Robnik and Berry, J. Phys. A 19, 669 (1986).

[17] B. S. Shastry, Phys. Rev. Lett. 69, 164 (1992).

[18] B. S. Shastry and B. Sutherland, Phys. Rev. Lett. 70, 4029 (1993).

\begin{tabular}{cc} 
RMT & CSM \\
\hline \hline$\pi \omega$ & $k_{F} r$ \\
$\pi^{2} x^{2} / 2$ & $-i k_{F}^{2} t$ \\
$1+k(\omega, x)$ & $\langle\rho(r, t) \rho(0,0)\rangle$ \\
$n(\omega, x)$ & $\int_{t}^{\infty}\left\langle j\left(r, t^{\prime}\right) j(0,0)\right\rangle d t^{\prime}$ \\
& \\
\hline \hline
\end{tabular}

TABLE I. Correspondence between random matrix theory (RMT) and Calogero-Sutherland model (CSM) 